\title{
Use of a Mindfulness Smartphone App in an Advanced Psychiatry Elective for Pharmacy Students
}

\section{J. Michael McGuire, PharmD, BCPP, Teresa Zhen, PharmD Candidate}

Belmont University College of Pharmacy

\begin{abstract}
Background: There is a mental health crisis among US University students. Evidence supporting techniques to help students manage the stress are critically needed. Mindfulness meditation is one such strategy which may be helpful for pharmacy students.

Innovation: Third-year pharmacy students enrolled in an Advanced Psychiatry elective course were invited to participate in the study. Students were given a 10-day assignment to participate in 10 free mindfulness sessions offered by the smart phone app, Headspace ${ }^{\circledR}$. The Mindful Attention Awareness Scale (MAAS) was completed before and after the assignment. Student qualitative feedback was also obtained after utilization of the app.

Findings: Seventy-six percent of students completed the mindfulness sessions daily. A small, non-statistically significant change in MAAS Total Score was observed in the students after use of the mindfulness app. The majority of students would recommend the mindfulness app to their patients, as well as $1^{\text {st }}$ year pharmacy students. The students also reported that the assignment aided in their education about non-pharmacologic treatment options for depression and anxiety.

Conclusion: This pilot study provides preliminary evidence for the further investigation of mindfulness meditation smartphone apps in pharmacy students both as a tool to aid in managing the stress of pharmacy school and as a teaching tool.
\end{abstract}

Keywords: mindfulness meditation, pharmacy students

\section{Description of the problem}

The headlines are startling - a mental health crisis facing American college students. Rates of depression among university students are nearly double the lifetime prevalence of depression in the United States. ${ }^{1,2}$ The prevalence among medical students was found to be $27 \%$ in a meta-analysis of 183 studies. $^{3} \quad$ Many would agree that the prevalence may be concerning in pharmacy students, as well.

Throughout pharmacy school, students learn not only about appropriate drug therapy for various disease states, but also about nonpharmacologic treatment options. One such nonpharmacologic treatment option for depression and anxiety is mindfulness meditation. Mindfulness meditation can be defined as "a way of being in which an individual maintains openness, patience and acceptance, while focusing attention on an unfolding situation in a nonjudgmental way" or, more simply, "a practice of non-judgmental awareness of present moment experiences". ${ }^{4,5}$ There is emerging evidence to support the benefit of mindfulness meditation for depression, anxiety, and burnout. ${ }^{4-5}$ Comprehensive training on mindfulness requires significant time, upwards of 30 hours, and a commitment to learn and implement the practices. ${ }^{4,6}$ There has been a rapid expansion of smartphone apps that promote themselves as leading participants through brief mindfulness meditation exercises. Mani and colleagues found that only a few of the apps they evaluated were actually deemed to engage in mindfulness meditation. ${ }^{7}$ Headspace ${ }^{\circledR}$ is a smartphone app

Corresponding author: J. Michael McGuire, PharmD, BCPP Associate Professor of Pharmacy Practice, Belmont University College of Pharmacy, 1900 Belmont Blvd, Nashville, TN 37212 Phone: 615-460-5723; Email: michael.mcguire@belmont.edu that guides participants through brief mindfulness sessions. Headspace ${ }^{\circledR}$ was found in a controlled trial to improve positive affect and reduce depression scores. ${ }^{5}$ Two studies utilized the Headspace ${ }^{\circledast}$ with physician trainees, medical students and residents, and both found that the trainees thought the app would be helpful for patients and would discuss mindfulness as a therapeutic option with their patients. ${ }^{6,8}$ Several studies have utilized mindfulness practices, not specifically Headspace ${ }^{\circledast}$, in graduate students and have found a benefit for academic stress, as well as resilience. ${ }^{9}$

It is important to differentiate mindfulness from mindset. Mindset is a different construct that may best be described as attitudes that affect learning and interactions with the world. ${ }^{10}$ Two mindsets that are important for educators are growth and fixed mindsets. A growth mindset believes that individuals can learn and adapt over time, developing new skills. A fixed mindset believes that individuals have certain abilities and talents that remain stable and there is limited opportunity for growth. A student's mindset could impact their willingness to try new skills, such as mindfulness meditation.

\section{Statement of the Innovation}

In order to teach pharmacy students about mindfulness meditation and to provide them with a potential exercise for themselves and their patients, a pilot study was conducted in an Advanced Psychiatry elective class to obtain quantitative effectiveness and qualitative feedback from the students on the use of the Headspace ${ }^{\circledR}$ app.

\section{The Innovation}

The pilot study was conducted at a private, faith-based university in the southeastern United States. The Advanced Psychiatry elective course for pharmacy students is taught in 
the spring semester of each academic year. Students may sign up for the elective class after they have received general training on mental health conditions in the fall semester of their third year. Each year, the course discusses a variety of topics related to mental health, including nonpharmacological/alternative treatment options for depression including psychotherapy, electroconvulsive therapy, transcranial magnetic stimulation, ketamine, and mindfulness meditation. When discussing depression in this class period, the students hear from an individual with a personal history of depression and a family history of suicide. Students were given an assignment to participate in the 10 free sessions offered by the Headspace ${ }^{\circledast}$ app around the class period, which discussed depression. Headspace ${ }^{\circledR}$ is a mindfulness meditation app that is available free for iPhone and Android devices. The moderator leads the participant through several exercises while the participant is seated in a comfortable, quiet space. Depending on the session that the participant is listening to, the exercises may include scanning the body, paying attention to the contact(s) between the chair and the body, counting breaths, specifically noting when the mind is wandering, and allowing the mind to freely wander. These exercises are designed to bring awareness to the individual's thoughts and the consistent practice allows the individual to focus more easily.

Students completed a pre- and post-survey, which included qualitative questions regarding their experience with the app and the Mindful Attention Awareness Scale (MAAS). ${ }^{11}$ The MAAS has been validated in general adults and college student population and is scored from 1 (almost always) to 6 (almost never) on a variety of statements related to mindfulness, such as unawareness of emotions, rushing through activities without awareness, forgetting names almost as soon as introduced, etc. The MAAS is scored by calculating a mean of the 15 items. A higher score on the MAAS indicates a higher level of dispositional mindfulness. The pre- and post-surveys were administered via Qualtrics to maintain participant anonymity. The study was reviewed by the Belmont University Institutional Review Board and determined exempt from Federal human subject research regulations. Although students were required to complete the mindfulness assignment, their participation in the surveys was voluntary.

Statistical analyses for the MAAS were conducted using SPSS ${ }^{\circ}$ Version 25 (IBM Corp, Armonk NY). Mean MAAS scores were compared using a two-tailed, paired student's t-test. Review of student responses to the post-survey questions that asked what worked well and what did not work well with the app was conducted by both of the authors and responses were sorted into categories.

\section{Findings}

Twenty-three students were enrolled in the Advanced Psychiatry elective course during the spring 2018 semester. Pre- and post-activity survey results are displayed in Table 1.
The results of the MAAS pre- and post- activity are shown in Table 2. The Cronbach's $\alpha$ for the MAAS pre- and post-activity were 0.842 and 0.824 , respectively.

In the post-activity survey, the students were asked two shortanswer questions regarding what worked well and what did not work well when using the app. There were several comments about the mindfulness sessions helping the students feel a sense of anxiety relief. One student commented:

"Setting time aside to be cognizant of my thoughts, emotions and even physicality (e.g. focused breathing) was very beneficial. Pharmacy students, like other doctoral students, develop a habit of being future-oriented due to deadlines and busy schedules. Having this mindset makes me numb to my current mental and physical status. Practicing mindfulness was therapeutic because it enabled me to process my emotions which created a sense of peace."

Several students specifically commented about how they enjoyed the app. They felt that the app was easy to use, they liked being able to set reminders to do sessions each day, and enjoyed some of the introductory videos that preceded some of the sessions. Several students commented that listening while in bed or at bedtime was helpful for them.

Regarding what did not work well, several students commented that they would forget to do the sessions despite having reminders set on their device. Only one student specifically stated that finding the time to do the sessions was a barrier. A number of students stated that they had a difficult time keeping their mind from wandering and focusing on either their body sensations or their breaths. One student specifically commented:

\footnotetext{
"The hardest part for me was focusing on my breaths and how my body felt. I would only be able to do it for a couple of minutes and would get distracted. I did notice that my focus got better with each exercise, but it's something I will keep working on."
}

The limitations of the study were the small sample size, duration of mindfulness practice, and potential bias from the respondents. Twenty-two students completed the pre-activity survey and 17 completed the post-activity survey. Extending the duration of the study past 10 days may produce a more significant change between the pre- and post- survey results. Students who were enrolled in the Advanced Psychiatry course may have been biased to find the practice of mindfulness more helpful than students not enrolled in the course. In addition, the students may have felt obligated to participate even though there was no course credit or bonus points offered for participation. 


\section{Critical Analysis}

Students reported that mindfulness meditation increased awareness of their emotions, surroundings, and daily tasks. Through this experience, students discovered an alternative practice that they can use for their own well-being, teach their patients, and suggest to colleagues. There was no statistically significant change in the MAAS mean score. However, many of the student's qualitative comments indicated that they felt the mindfulness exercise was a helpful activity for their well-being. Additionally, all students who completed the mindfulness app exercise felt that the tool may benefit patients, as well as other pharmacy students. A shared challenge amongst students in utilizing any new tool was remembering to practice it each day.

Our findings are consistent those of Taylor and colleagues, ${ }^{6}$ who used the Headspace ${ }^{\circledast}$ app with resident physicians. Like our study, the residents were asked to use the 10 free sessions offered by Headspace ${ }^{\circledast}$. The residents completed the MAAS, as well as the Maslach Burnout Inventory (MBI), prior to $(n=33)$ and after $(n=11)$ utilizing the app for 10 days. The authors reported a statistically significant change in the percentage of residents who would recommend the app to their patients (48\% to $61 \%$ ), which is consistent with our findings. There was no statistically significant change in the MBI and the MAAS outcomes were not reported. Similar to our students' comments on time or remembering, eighty-four percent of participants in the Taylor study indicated that lack of time and knowledge were the primary barriers to regular meditation practice.

Yang and colleagues utilized the Headspace ${ }^{\circledR}$ app with medical students. ${ }^{8}$ Participants $(n=45)$ completed 3 surveys that included demographic questions, as well as the Perceived Stress Scale (PSS), Five-Facet Mindfulness Questionnaire (FFMQ) and the General Well-Being Schedule (GWBS) at baseline (T1), 1 month (T2) and 2 months (T3). Students in the intervention group were asked to use the app daily or as much as possible for 30 days. The mean number of days used was 11.97 during the 30 days. There was a statistically significant decrease in the PSS and increase in GWBS compared to a control group. Only the observing subscale of the FFMQ was significantly different from controls. Only sixty percent of the intervention group ( $n=27$ ) used the app at least once during the 30 days although $98 \%$ of all participants were in a state of distress at baseline according to the General Well Being Schedule. Similar to Taylor and consistent with comments in our study, students struggled with implementing mindfulness consistently.

\section{Key Issues}

Key issues identified in our study and by Taylor ${ }^{6}$, and Yang ${ }^{8}$ are that despite what trainees may feel about mindfulness or recommend to patients, time is a reported barrier even though the Headspace ${ }^{\circledR}$ sessions are very brief. Although students are stressed and recognize the potential utility of mindfulness meditation, a significant number will not commit the time to daily practice the exercise. This is a key issue even though applications like Headspace ${ }^{\circledR}$ offer brief interventions with emerging data of their utility in managing academic stress.

\section{Next Steps}

Future studies with mindfulness meditation apps in pharmacy students should collect additional quantitative results on levels of anxiety and examine course performance. Studies using the MAAS, in addition to the use of specific rating scales that assess student anxiety, such as the Smith Anxiety Scale, the Perceived Stress Scale and the Maslach Burnout Inventory ${ }^{4}$ are warranted. Additional studies on other strategies to manage stress and anxiety among pharmacy students are also needed.

\section{Acknowledgements: None \\ Funding/Support: None}

Conflicts of Interest: Dr. McGuire has received honorarium or consultant fees from Sunovion Pharmaceuticals, Alkermes Inc, and Otsuka America Pharmaceuticals Inc. Dr. McGuire also holds stock in Bristol-Myers Squibb Inc. Teresa Zhen has no conflicts of interest to report.

\section{References}

1. Ibrahim AK, Kelly SJ, Adams CE, Glazebrook C. A systematic review of studies of depression prevalence in university students. J Psychiatr Res. 2013;47(3):391-400. doi: 10.1016/j.jpsychires.2012.11.015.

2. Kessler RC, Berglund P, Demler O, et al. The epidemiology of major depressive disorder: results from the National Comorbidity Survey Replication (NCS-R). JAMA. 2003;289(23):3095-3105.

3. Rotenstein LS, Ramos MA, Torre M, et al. Prevalence of depression, depressive symptoms, and suicidal ideation among medical students: A systematic review and meta-analysis. JAMA. 2016;316(21):2214-2236. doi: 10.1001/jama.2016.17324.

4. Gilmartin H, Goyal A, Hamati MC, Mann J, Saint S, Chopra V. Brief mindfulness practices for healthcare providers - A systematic literature review. Am J Med. 2017;130(10): 1219.e11219.e17. doi: 10.1016/j.amjmed.2017.05.041.

5. Howells A, Ivtzan I, Eiroa-Orosa FJ. Putting the 'app' in happiness: A randomised controlled trial of a smartphone-based mindfulness intervention to enhance wellbeing. J Happiness Stud. 2016;17(1):163-185

6. Taylor M, Hageman JR, Brown M. A mindfulness intervention for residents: Relevance for pediatricians. Pediatr Ann. 2016;45(10):e373-e376. doi: 10.3928/19382359-20160912-01. 
7. Mani M, Kavanagh DJ, Hides L, Stoyanov SR. Review and evaluation of mindfulness-based iPhone apps. JMIR Mhealth Uhealth.

2015;3(3):e82. doi: 10.2196/mhealth.4328.

8. Yang E, Schamber E, Meyer RML, Gold JI. Happier healers: Randomized controlled trial of mobile mindfulness for stress management. J Altern Complement Med. 2018;24(5):505-513. doi: 10.1089/acm.2015.0301.

9. Kelly M. Does mindfulness practice improve the mental health and wellbeing of healthcare students? J Psychiatr Ment Health Nurs. 2017;24(1):84-89. doi: 10.1111/jpm.12348.

10. Cooley JH, Larson S. Promoting a growth mindset in pharmacy educators and students. Curr Pharm Teach Learn. 2018;10(6):675-679. doi: 10.1016/j.cptl.2018.03.021.

11. Brown KW, Ryan RM. The benefits of being present: mindfulness and its role in psychological well-being. J Pers Soc Psychol. 2003;84(4):822-848. 
Table 1. Pre and Post-activity Questions

\begin{tabular}{|c|c|c|}
\hline Pre-Survey Question ( $n=22)$ & Yes & No \\
\hline I have used a mindfulness app or program in the past. & 2 & 20 \\
\hline \multicolumn{3}{|l|}{ Post-Survey Questions ( $n=17)$} \\
\hline Did you practice the mindfulness sessions daily? & 13 & 4 \\
\hline I think this would be a helpful tool for my patients. & 17 & 0 \\
\hline I would recommend the Headspace ${ }^{\circledR}$ app to patients. & 17 & 0 \\
\hline I think this would be a helpful tool for my colleagues. & 17 & 0 \\
\hline
\end{tabular}


Table 2. Change in Mindful Attention Awareness Scale (MAAS) ${ }^{\text {a }}$ Scores

Pre-Activity

3.34

Mean MAAS Total Score

Mean MAAS Score on Individual Items

I could be experiencing some emotion and not be conscious of it until sometime later.

I break or spill things because of carelessness, not paying attention, or thinking of something else.

I find it difficult to stay focused on what's happening in the present.

I tend to walk quickly to get where I'm going without paying attention to what I experience along the way.

I tend not to notice feelings of physical tension or discomfort until they really grab my attention.

I forget a person's name almost as soon as I've been told it for the first time.

It seems I am "running on automatic," without much awareness of what I'm doing.

I rush through activities without being really attentive to them.

I get so focused on the goal I want to achieve that I lose touch with what I'm doing

right now to get there.

I do jobs or tasks automatically, without being aware of what I'm doing.

I find myself listening to someone with one ear, doing something else at the same time.

I drive places on 'automatic pilot' and then wonder why I went there.

I find myself preoccupied with the future or the past.

I find myself doing things without paying attention.

I snack without being aware that I'm eating.
3.60

0.186
3.73

Post-Activity

p-value

4.32

0.551

4.32

4.29

0.661

3.05

3.53

0.188

2.45

3.06

0.181

3.73

4.12

0.262

2.41

3.24

0.020

3.41

3.31

1.000

3.5

3.94

0.130

3.09

3.06

0.773

3.14

3.65

0.163

2.86

3.41

0.132

4.05

2.64

4.06

0.735

3.18

3.12

0.501

4.5

3.35

0.463

4.53

0.910

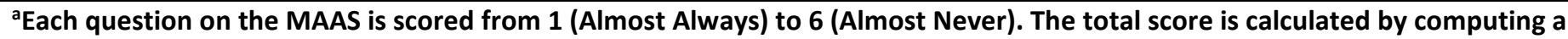
mean of the 15 items. A higher score reflects a higher degree of mindfulness. 